\title{
Composição e riqueza de espécies de uma comunidade de Coleoptera (Insecta) na Caatinga
}

\author{
Rozileudo da Silva Guedes' (1D, Fernando César Vieira Zanella² (i) \& Paschoal Coelho Grossi ${ }^{3}$ (1)
}

\author{
1. Unidade Acadêmica de Engenharia Florestal, Universidade Federal de Campina Grande, Av. Universitária, s/n, Santa Cecilia, 58708-1 10 Patos, PB, Brasil. \\ (rozileudo@gmail.com) \\ 2. Pós-Graduação em Biodiversidade Neotropical, Universidade Federal da Integração Latino-Americana, Av. Tarquinio Joslin dos Santos, 1000, Jardim Universitário, \\ 85867-000 Foz do Iguaçu, PR, Brasil. (fcvzanella@gmail.com) \\ 3. Programa de Pós-Graduação em Entomologia Agrícola, Departamento de Agronomia/ Fitossanidade, Universidade Federal Rural de Pernambuco, Rua Manuel \\ Medeiros, s/n, Dois Irmãos, 52171-900 Recife, PE, Brasil. (paschoal.grossi@gmail.com)
}

RESUMO. Os besouros apresentam grande diversidade taxonômica e funcional, no entanto, para a Região Nordeste, especialmente para a porção com vegetação de Caatinga, o conhecimento da fauna de Coleoptera é muito restrito. Por se tratar de uma região com grande diversidade de paisagens, tornase necessário conhecer a coleopterofauna que ocorre nas diferentes fitofisionomias, buscando com isso abranger a variabilidade espacial encontrada nessa região xérica. Diante disso, o objetivo deste estudo foi realizar um levantamento das espécies de besouros no Semiárido paraibano. As coletas foram realizadas semanalmente durante quatro anos (2009-2013) utilizando uma armadilha Malaise por fitofisionomia, sendo amostrada uma área com vegetação xerófila e outra com mata ciliar. Foram coletados 6.567 indivíduos pertencentes a 383 espécies e 42 famílias de Coleoptera. Chrysomelidae (78 spp.), Curculionidae (57 spp.) e Cerambycidae (28 spp.) foram as famílias mais ricas. Em relação às mais abundantes, Chrysomelidae (30,4\% dos indivíduos), Curculionidae $(8,3 \%)$ e Tenebrionidae $(7,7 \%)$ se destacaram. Foi verificado um elevado número de singletons e doubletons, apesar do prolongado período amostral, indicando que deve haver ainda muitas espécies a serem registradas na área de estudos. Nossos resultados demonstram uma rica fauna de besouros na região semiárida estudada e fornece uma lista de besouros de ocorrência no Semiárido paraibano, com novos registros de espécies para o estado e contribui assim com o conhecimento da diversidade de Coleoptera no Nordeste do Brasil.

PALAVRAS-CHAVE. Besouros, riqueza de espécies, Malaise, Semiárido.

ABSTRACT. Composition and species richness of the community of Coleoptera (Insecta) in the Caatinga. The beetles present great taxonomic and functional diversity; however, in the Northeast region of Brazil, especially in the semiarid Caatinga region, the knowledge on the Coleoptera fauna is very restricted. Because the Caatinga presents different landscapes, it is important to survey the coleopterofauna in the different phytophysiognomies, seeking to encompass a spatial variability found in this xeric region. Therefore, the objective of this study was to perform a survey of beetles in the semiarid region of Paraíba state, Northeast Brazil. We collected weekly during four years (2009-2013) using Malaise traps in two phytophysiognomies, an area with xerophytic vegetation and another with riparian forest. A total of 6,567 individuals belonging to 383 species and 42 families of Coleoptera were sampled. Chrysomelidae (78 spp.), Curculionidae (57 spp.), and Cerambycidae (28 spp.) had a higher richness, while Chrysomelidae (30.4\% of individuals), Curculionidae (8.3\%), and Tenebrionidae (7.7\%) were the most abundant. A large number of singletons and doubletons were collected despite the long sampling period, indicating that there must be much more species to be recorded. Our results demonstrate a rich fauna of beetles in the studied region. We provide a list of occurring beetles with new records of species for the Paraíba state and thus contribute to the knowledge of the diversity of the Coleoptera in the Northeast Brazil.

KEYWORDS. Beetles, species richness, Malaise, Semiarid.

Os insetos representam o mais diversificado e bemsucedido grupo de animais terrestres e, dentre estes, a ordem Coleoptera se destaca pela grande diversidade taxonômica e funcional (BouCHARD, 2014). No Brasil, são conhecidas mais de 32 mil espécies distribuídas em 114 famílias (MONNÉ \& CosTA, 2019).

Os besouros ocupam inúmeros nichos ecológicos (GANHO \& MARINONI, 2006; CASARI \& IDE, 2012; Gullan \& CRANSTON, 2014), alimentando-se de todos os tipos de materiais vegetais e animais, sendo geralmente fitófagos, porém, outros são predadores, fungívoros, detritívoros e alguns poucos parasitas (MARINONI et al., 2001; TRIPLEHORN \& JOHNSON, 2011). Essa variabilidade de hábitos alimentares faz com que os besouros desempenhem papéis importantes nos ecossistemas terrestres onde se encontram, atuando em diversos processos ecológicos fundamentais para o funcionamento desses ambientes, incluindo auxílio na decomposição de matéria orgânica, manutenção da estrutura do solo, polinização, dispersão de sementes e autorregularão das populações, incluindo as de artrópodes fitófagos potencialmente pragas (GULLAN \& CRAnston, 2012). 
Apesar do sucesso evolutivo do grupo e da importância ecológica e econômica, além de uma provável fauna endêmica para a Caatinga, o conhecimento relativo da mesma é ainda muito limitado e desuniforme dependendo do táxon considerado, sendo escassas as informações sobre os besouros desse bioma (BRANDÃo \& YAMAMOTO, 2003). Para o Nordeste brasileiro, poucos são os trabalhos encontrados na literatura com Coleoptera. As pesquisas têm se concentrado em amostragens com a utilização de armadilhas Malaise (IANNUZZI et al., 2003; MAIA et al., 2003; IANNUZZI et al., 2006), armadilhas de solo, Malaise e guarda-chuva entomológico (VASCONCELlos et al., 2010), armadilhas de luz (modelo Luiz de Queiroz), rede entomológica e/ou armadilhas com iscas para Cerambycidae (NASCimento \& Bravo, 2014; NASCimEnto et al., 2016) e com iscas para escarabeídeos (HERNÁNDEZ, 2005, 2007; LOPES \& LOUZADA, 2005; LOPES et al., 2006; Liberal et $a l .$, 2011; SAlomão et al., 2014; SAlomão \& IANNUZZI, 2015; Silva et al., 2017; VieIRA et al., 2017). No entanto, tais pesquisas são restritas a poucas famílias de Coleoptera. Há registro de apenas um artigo abordando coleópteros de mata ciliar na região do Xingó, entre os estados de Alagoas e Sergipe, o que evidencia a necessidade de conhecer a fauna desse ambiente (IANNUZzı et al., 2003). A limitação de conhecimento da coleopterofauna faz com que áreas representativas de regiões da Caatinga não tenham sido ainda estudadas, a exemplo da região do Seridó paraibano. Para o estado da Paraíba, o volume de pesquisas com Coleoptera é incipiente; destacam-se ENDRES et al. (2005, 2007), Hernández $(2005,2007)$ para Scarabaeidae, e de VASCONCELlos et al. (2010) para besouros em geral.

A Caatinga destaca-se por ser o único bioma exclusivamente brasileiro, ocupando uma área de pouco mais que $912.000 \mathrm{~km}^{2}$ do território (SILVA et al., 2017). É composta por um mosaico de florestas secas e vegetação arbustiva (savana-estépica), com enclaves de florestas úmidas montanas e de Cerrado (TABARELli \& Silva, 2003). $\mathrm{Na}$ depressão Sertaneja Setentrional, uma das ecorregiões propostas para a Caatinga por VelLoso et al. (2002), as florestas com vegetação xerófila e as matas ciliares são os principais ambientes.

A área florestal remanescente do bioma está altamente fragmentada, o que leva à perda de hábitat, e grande parte de seus ecossistemas apresentam alterações por ações antrópicas (CASTElletti et al., 2003; Leal et al., 2005; Santos et al., 2011). Aliado a isso, a Caatinga é considerada a área mais crítica em relação ao desconhecimento da composição faunística no país (CALOR \& BRAVO, 2014). Soma-se a isso o fato da depressão Sertaneja Setentrional ser a ecorregião mais ameaçada do bioma em termos de conservação das espécies (Velloso et al., 2002). Assim, a urgência em se conhecer a biodiversidade desse bioma é fundamental e necessária (Lewinsohn, 2000). Para isso, o estudo da comunidade de besouros na Caatinga e em particular na área estudada, por se tratar de uma localidade classificada como de elevado interesse e indicada como prioritária para a conservação da biodiversidade da Caatinga (SiLva et al., 2003), é essencial na redução dessa lacuna de conhecimento da biodiversidade desse bioma

Diante do exposto, o objetivo deste estudo foi realizar um levantamento das espécies de besouros no Semiárido da Paraíba visando contribuir para o conhecimento da biodiversidade de Coleoptera da Caatinga e da Região Nordeste.

\section{MATERIAL E MÉTODOS}

Caracterização da área de estudo. As coletas foram realizadas na Fazenda Tamanduá, localizada no município de Santa Terezinha, Estado da Paraíba, região Semiárida do Nordeste $\left(37^{\circ} 24^{\prime} 22,5^{\prime \prime} \mathrm{O} ; 07^{\circ} 01^{\prime} 30,3{ }^{\prime \prime} \mathrm{S}\right)$. A propriedade possui cerca de 3.000 ha, com duas áreas destinadas à preservação, sendo uma categorizada como Reserva Particular do Patrimônio Natural (RPPN), com cerca de 900 ha, e outra de Reserva Legal, com aproximadamente 325 hectares, que há pouco mais de 30 anos não sofre pressão antrópica.

De acordo com o sistema de Köppen, o clima é classificado como BSh (semiárido) (Álvvares et al., 2013). A área da fazenda está inserida na ecorregião da Caatinga denominada Depressão Sertaneja Setentrional (VelLoso et al., 2002), caracterizada por um clima quente semiárido, com temperaturas médias anuais altas estimadas entre 26 a $28^{\circ} \mathrm{C}$ (NIMER, 1972), com precipitação média de 500 a $800 \mathrm{~mm}$ anuais e dois períodos pluviométricos distintos: o chuvoso (ocorrendo nos primeiros meses do ano) e o seco (segundo semestre do ano, podendo ser mais longo) (VELLOSO et al., 2002). A vegetação da região, localmente denominada de Seridó, é do tipo Caatinga arbustivo-arbórea aberta e esparsa, com registro de espécies como Amburana cearensis (Allemão) A.C. Sm (Fabaceae), Spondias tuberosa Arruda (Anacardiaceae), Aspidosperma pyrifolium Mart. (Apocynaceae) e um componente arbustivo de Caesalpinia spp. (Fabaceae), Mimosa spp. (Fabaceae), Jatropha spp. (Euphorbiaceae), e Acacia spp. (Fabaceae) (Prado, 2003).

$\mathrm{O}$ remanescente de vegetação xerófila estudado fica em um fragmento da Reserva Legal da propriedade e apresenta um estrato arbustivo-arbóreo heterogêneo, com predomínio de catingueira [Poincianella pyramidalis (Tul.) L. P. Queiroz - Fabaceae], imburana de cambão [Commiphora leptophloeos (Mart.) J.B. Gillett - Burseraceae], rompe-gibão (Erythroxylum pungens O. E. Schulz - Erythroxylaceae), jurema branca [Piptadenia stipulacea (Benth.) Ducke - Fabaceae] e pereiro (Aspidosperma pyrifolium Mart. Apocynaceae) e um componente herbáceo abundante durante o período chuvoso (GUEDEs et al., 2012), especialmente nas áreas de clareiras naturais e antrópicas. No entanto, apesar de ser relativamente bem preservada no contexto da região, a vegetação apresenta evidências de que houve exploração seletiva de espécies arbóreas. A vegetação decídua dessa área fica quase totalmente sem folhas por vários meses durante $o$ período de estiagem, com exceção das perenifólias e daquelas que ocorrem em locais com acúmulo de água no solo.

A área de mata ciliar margeia um rio intermitente da região que seca durante o período de estiagem, apresentando espécies de mata higrófila, destacando-se Licania rigida 
Benth (oiticica, Chrysobalanaceae) e solos mais profundos em relação à área com vegetação xerófila. Esse tipo de ambiente é capaz de manter uma vegetação sempre verde, mesmo durante a estação seca, devido à disponibilidade hídrica do solo (Prado, 2003). A área ciliar fica próxima de culturas agrícolas irrigadas, o que é um cenário comum na região, pois são áreas com solos mais profundos, com menor estresse hídrico e mais exploradas pelo homem para essa prática. $\mathrm{Na}$ propriedade estudada se pratica agricultura orgânica, portanto, não faz uso de agrotóxicos e próximo à mata ciliar há áreas de pastagem irrigada e plantios orgânicos de mangueiras, meloeiros e de melancia.

Metodologia de amostragem. As coletas foram realizadas semanalmente de novembro de 2009 a outubro de 2013 em duas fitofisionomias de Caatinga com utilização simultânea e ininterrupta de uma armadilha do tipo Malaise em cada área. A armadilha é constituída por uma tenda de náilon, suspensa por hastes de ferro, composta por tecido preto transparente que desce da parte dorsal no centro da armadilha até o solo, com dimensões de $1,70 \mathrm{~m}$ de comprimento, 1,50 m de altura na parte da frente e 0,90 $\mathrm{m}$ na parte posterior. As armadilhas foram instaladas de forma que o tecido interceptador ficasse perpendicular ao sentido Leste-Oeste, com o maior comprimento paralelo ao sentido Norte-Sul, com o frasco coletor voltado para o Norte. No frasco coletor, que consistiu de um recipiente plástico adaptado, foi preenchido com álcool etílico hidratado 92,8 INPM, para conservar os insetos coletados. Esse tipo de armadilha não utiliza atrativo, interceptando os insetos durante o voo, continuamente. O material foi retirado e o álcool devidamente reposto. Os exemplares obtidos foram acondicionados em frascos contendo álcool $70 \%$, posteriormente triados, montados em alfinetes entomológicos e etiquetados. Os espécimes estão depositados nas coleções dos laboratórios de Taxonomia de Insetos da Universidade Federal Rural de Pernambuco, Campus de Recife (CERPE), Pernambuco, e de Entomologia Florestal da Universidade Federal de Campina Grande (UFCG), Campus de Patos, Paraíba.

As duas fitofisionomias estudadas integram uma área com vegetação xerófila e outra ciliar. Na primeira a armadilha Malaise foi colocada próxima à borda e, na segunda ao lado da floresta, em área aberta. Em cada área amostrada a armadilha revezava entre três pontos amostrais a cada quatro semanas. Nessa mesma ocasião, também havia a troca entre as áreas, a fim de evitar a influência da eficiência diferencial na amostragem devido, por ventura, ao equipamento de coleta.

Identificação dos táxons. Os coleópteros foram triados em microscópio estereoscópico e reconhecidos até o nível de família utilizando bibliografia especializada (Booth et al., 1990; Arnett \& Thomas, 2001; ArnetT et al., 2002; Constantino et al., 2002; Bouchard et al., 2011; TRIPLEHORN \& Johnson, 2011; CASARI \& IDE, 2012). Para a classificação supragenérica, utilizou-se a organização taxonômica adotada por Bouchard et al. (2011). Para a identificação em níveis hierárquicos mais inferiores, contouse com a colaboração de especialistas.
Análise dos dados. Padrões de estrutura e composição da comunidade de besouros foram descritos utilizando-se os parâmetros de riqueza e abundância. A curva de acumulação de espécies por meio de rarefação foi estimada, considerando o total das duas fitofisionomias (Gotelli \& Cowell, 2001). Para estimar a riqueza de espécies foram utilizados Chao1, Jackknife1, Jackknife2 e Bootstrap. Para as análises de suficiência amostral e dos estimadores de riqueza, utilizou-se o pacote vegan (OKSANEN et al., 2016) por meio do programa R (R CoRe Team, 2018).

\section{RESULTADOS}

A fauna de besouros amostrada foi representada por 42 famílias distribuídas em 383 espécies e um total de 6.567 indivíduos (Tab. I). Chrysomelidae foi a família mais rica com 78 espécies, seguida por Curculionidae (57), Cerambycidae (28), Elateridae (27) e Staphylinidae (26 espécies); em conjunto, essas famílias foram responsáveis por $57,6 \%$ do total. Das famílias registradas, grande parte foi representada por apenas uma ou duas espécies, o que correspondeu a 47,6\% do total de espécies amostradas.

Em termos de abundância, Chrysomelidae também se destacou correspondendo a 30,4\% dos indivíduos amostrados, seguida por Curculionidae, Tenebrionidae, Mordellidae e Elateridae, e em conjunto foram responsáveis por $60,3 \%$ (3.963 exemplares) do total de indivíduos amostrados (Tab. I).

Os crisomelídeos foram representados por 53 gêneros e, dentre as subfamílias amostradas, Galerucinae se destacou com o maior número de espécies (24), seguida por Bruchinae (19) e Eumolpinae (14). Bruchinae ( $\mathrm{N}=1.379$; $69 \%)$, Cryptocephalinae $(\mathrm{N}=168 ; 8,4 \%)$, Chysomelinae $(\mathrm{N}=140 ; 7,0 \%)$ e Galerucinae $(\mathrm{N}=135 ; 6,8 \%)$ foram as mais abundantes.

A curva de acumulação de espécies não mostrou tendência de estabilização (Fig. 1). O número de espécies

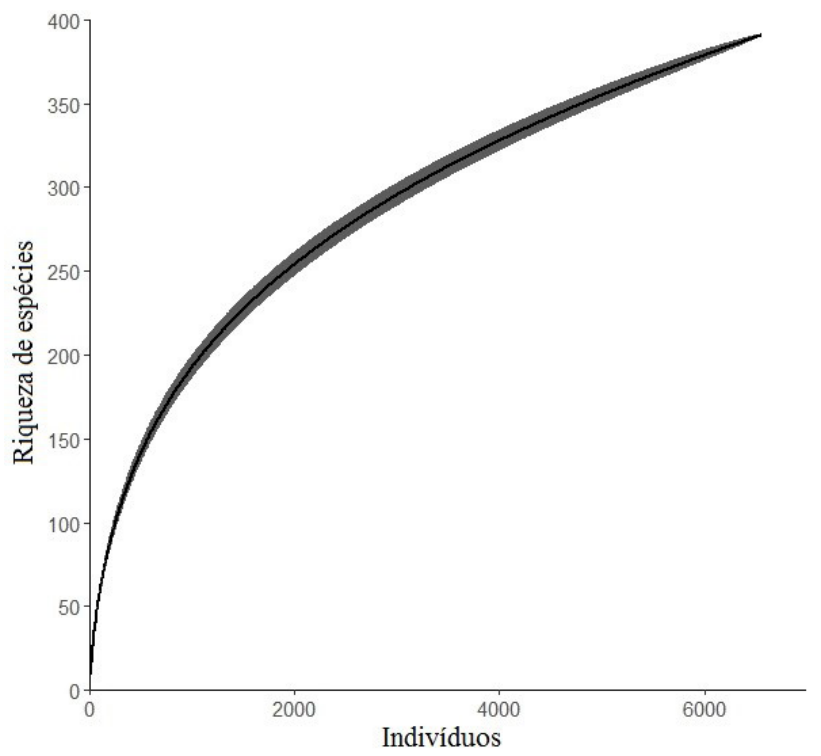

Fig. 1. Representação da curva de acumulação de espécies e do intervalo de confiança $(95 \%)$ da comunidade de besouros coletada em área de mata ciliar e vegetação xerófila de caatinga, capturadas com armadilha Malaise de novembro de 2009 a outubro de 2013, Santa Terezinha, Paraíba, Brasil. 
Tab. I. Número de espécies e de indivíduos coletados com armadilha Malaise em duas fitofisionomias de Caatinga - mata ciliar (MC) e vegetação xerófila (VX) -, e seus respectivos totais, por família de Coleoptera, no período de novembro de 2009 a outubro de 2013, Santa Terezinha, Paraíba, Brasil.

\begin{tabular}{|c|c|c|c|c|c|c|}
\hline \multirow{2}{*}{ Famílias } & \multicolumn{3}{|c|}{$\mathrm{N}^{\mathrm{o}}$ de espécies } & \multicolumn{3}{|c|}{$\mathrm{N}^{\circ}$ de indivíduos } \\
\hline & $\mathrm{MC}$ & VX & Total & $\mathrm{MC}$ & VX & Total \\
\hline Chrysomelidae & 70 & 39 & 78 & 1.300 & 699 & 1.999 \\
\hline Curculionidae & 48 & 32 & 57 & 327 & 215 & 542 \\
\hline Cerambycidae & 21 & 21 & 28 & 143 & 74 & 217 \\
\hline Elateridae & 25 & 18 & 27 & 352 & 81 & 433 \\
\hline Staphylinidae & 20 & 6 & 26 & 60 & 9 & 69 \\
\hline Carabidae & 24 & 9 & 25 & 66 & 43 & 109 \\
\hline Cocccinellidae & 14 & 9 & 14 & 313 & 63 & 376 \\
\hline Tenebrionidae & 14 & 5 & 15 & 437 & 68 & 505 \\
\hline Mordellidae & 9 & 10 & 11 & 200 & 284 & 484 \\
\hline Buprestidae & 7 & 7 & 11 & 42 & 31 & 73 \\
\hline Nitidulidae & 9 & 6 & 9 & 33 & 9 & 42 \\
\hline Cleridae & 6 & 7 & 8 & 273 & 156 & 429 \\
\hline Scarabaeidae & 6 & 5 & 9 & 10 & 23 & 33 \\
\hline Ptinidae & 6 & 7 & 8 & 110 & 29 & 139 \\
\hline Phalacridae & 2 & 2 & 2 & 183 & 71 & 254 \\
\hline Anthicidae & 5 & 2 & 5 & 122 & 2 & 124 \\
\hline Aderidae & 3 & 1 & 3 & 19 & 9 & 28 \\
\hline Bostrichidae & 3 & 3 & 3 & 79 & 89 & 168 \\
\hline Phengodidae & 4 & 4 & 4 & 42 & 66 & 108 \\
\hline Lampyridae & 4 & 1 & 4 & 97 & 3 & 100 \\
\hline Meloidae & 2 & 2 & 4 & 4 & 2 & 6 \\
\hline Trogossitidae & 3 & 2 & 3 & 3 & 2 & 5 \\
\hline Ptilodactylidae & 1 & - & 1 & 7 & - & 7 \\
\hline Silvanidae & 2 & - & 2 & 3 & - & 3 \\
\hline Zopheridae & 4 & 3 & 5 & 72 & 65 & 137 \\
\hline Dermestidae & 2 & 2 & 2 & 11 & 5 & 16 \\
\hline Leiodidae & 1 & 1 & 1 & 2 & 5 & 7 \\
\hline Anthribidae & 1 & 1 & 1 & 23 & 23 & 46 \\
\hline Biphyllidae & 1 & - & 1 & 1 & - & 1 \\
\hline Brentidae & 2 & - & 2 & 2 & - & 2 \\
\hline Cantharidae & 1 & - & 1 & 3 & - & 3 \\
\hline Chelonariidae & - & 1 & 1 & - & 5 & 5 \\
\hline Corylophidae & 1 & 1 & 2 & 1 & 2 & 3 \\
\hline Dytiscidae & - & 1 & 1 & - & 1 & 1 \\
\hline Endomychidae & - & 1 & 1 & - & 1 & 1 \\
\hline Scraptiidae & 1 & - & 1 & 8 & - & 8 \\
\hline Histeridae & 1 & - & 1 & 1 & - & 1 \\
\hline Latridiidae & 1 & 1 & 1 & 5 & 1 & 6 \\
\hline Melyridae & 1 & 1 & 1 & 1 & 2 & 3 \\
\hline Oedemeridae & 2 & 2 & 2 & 11 & 16 & 27 \\
\hline Scirtidae & 1 & 1 & 1 & 29 & 1 & 30 \\
\hline Throscidae & 1 & - & 1 & 17 & - & 17 \\
\hline Total & 329 & 214 & 383 & 4.412 & 2.155 & 6.567 \\
\hline
\end{tabular}

estimado variou entre os quatro estimadores utilizados. A estimativa Bootstrap foi a menor com 443 espécies e a Jackknife2 a maior, com 608 táxons. Jackknife1 e Chaol foram intermediários, com 523 e 554 espécies, respectivamente. Assim, o número de espécies amostradas variou entre 62,9 a $73,2 \%$ da quantidade estimada para a área.

As espécies mais abundantes foram Acanthoscelides sp. 1 (Chrysomelidae), correspondendo a $5,54 \%$ do total de indivíduos, Epitragus (Semilepitragus) sp. 2 (Tenebrionidae, 5,02\%), Zabrotes sp. 2 (Chrysomelidae, 3,72\%), Acylomus sp. (Phalacridae, 3,33\%), Corinthiscus sp. 1 (Cleridae, 2,91\%) e Zabrotes sp. 1. (Chrysomelidae) com $2,91 \%$ do total (Tab. II). Das 383 espécies amostradas, 129 foram categorizadas como singletons, o que representa $33,7 \%$ do total, e 49 como doubletons $(12,8 \%)$. 
Tab. II. Lista de espécies de Coleoptera coletadas em Caatinga com respectivas abundâncias totais e para as duas fitofisionomias - mata ciliar (MC) e vegetação xerófila (VX) -, coletadas com armadilha Malaise no período de de novembro de 2009 a outubro de 2013, Santa Terezinha, Paraíba.

\begin{tabular}{|c|c|c|c|c|}
\hline $\begin{array}{l}\text { FAMÍLIA } \\
\text { Subfamília }\end{array}$ & Espécie/morfoespécie & $\mathrm{MC}$ & vX & Total \\
\hline \multicolumn{5}{|l|}{ ADERIDAE } \\
\hline & Aderus sp. 1 & 14 & 9 & 23 \\
\hline & Aderus sp. 2 & 4 & & 4 \\
\hline & Aderus sp. 3 & 1 & & 1 \\
\hline \multicolumn{5}{|l|}{ ANTHICIDAE } \\
\hline \multirow[t]{5}{*}{ Anthicinae } & Acanthinus sp. & 109 & 1 & 110 \\
\hline & Anthicus sp. & 6 & & 6 \\
\hline & Acanthinus sp. & 1 & & 1 \\
\hline & Formicilla sp. 1 & 2 & & 2 \\
\hline & Formicilla sp. 2 & 4 & 1 & 5 \\
\hline \multicolumn{5}{|l|}{ ANTHRIBIDAE } \\
\hline Anthribinae & Phaenithon sp. & 23 & 23 & 46 \\
\hline \multicolumn{5}{|l|}{ BIPHYLLIDAE } \\
\hline & Diplocoelus sp. & 1 & & 1 \\
\hline \multicolumn{5}{|l|}{ BOSTRICHIDAE } \\
\hline \multirow[t]{3}{*}{ Bostrichinae } & Bostrichopsis sp. & 16 & 5 & 21 \\
\hline & Tetrapriocera defracta Lesne, 1901 & 11 & 22 & 33 \\
\hline & Xyloperthella picea (Olivier, 1790) & 52 & 62 & 114 \\
\hline \multicolumn{5}{|l|}{ BRENTIDAE } \\
\hline \multirow[t]{2}{*}{ Apioninae } & Apion sp. 1 & 1 & & 1 \\
\hline & Apion sp. 2 & 1 & & 1 \\
\hline \multicolumn{5}{|l|}{ BUPRESTIDAE } \\
\hline \multirow[t]{8}{*}{ Agrinae } & Agrilus sp. 1 & 1 & 1 & 2 \\
\hline & Agrilus sp. 2 & & 12 & 12 \\
\hline & Agrilus sp. 3 & 17 & & 17 \\
\hline & Agrilus sp. 4 & & 1 & 1 \\
\hline & Agrilus sp. 5 & 1 & & 1 \\
\hline & Pachyschelus sp. & 1 & & 1 \\
\hline & Taphrocerus sp. 1 & 7 & 2 & 9 \\
\hline & Taphrocerus sp. 2 & 1 & & 1 \\
\hline \multirow{3}{*}{ Buprestinae } & Chrysobothris sp. 1 & 14 & 13 & 27 \\
\hline & Chrysobothris sp. 2 & & 1 & 1 \\
\hline & Tetragonoschema sp. & & 1 & 1 \\
\hline \multicolumn{5}{|l|}{ CANTHARIDAE } \\
\hline Chauliognathinae & Chauliognathus sp. & 3 & & 3 \\
\hline \multicolumn{5}{|l|}{ CARABIDAE } \\
\hline Carabinae & Calosoma (Castrida) retusum (Fabricius, 1775) & 2 & 1 & 3 \\
\hline Cicindelinae & Brasiella misella (Chaudoir, 1854) & 4 & 4 & 8 \\
\hline Harpalinae & Agra sp. & 1 & & 1 \\
\hline & Anchonoderus sp. & 1 & & 1 \\
\hline & Calleida (Calleida) sp. 1 & 1 & 3 & 4 \\
\hline & Calleida (Calleida) sp. 2 & 1 & & 1 \\
\hline & Calleida (Calleida) sp. 3 & & 1 & 1 \\
\hline & Calleida (Calleida) sp. 4 & 3 & 1 & 4 \\
\hline & Calleida (Calleida) sp. 5 & 10 & 3 & 13 \\
\hline & Colliuris sp. & 4 & 1 & 5 \\
\hline & Lebia (Lebia) sp. 1 & 2 & & 2 \\
\hline & Lebia (Lebia) sp. 2 & 1 & & 1 \\
\hline & Lebia (Lebia) sp. 3 & 1 & & 1 \\
\hline & Lebia (Lebia) sp. 4 & 6 & & 6 \\
\hline & Leptotrachelus sp. & 3 & & 3 \\
\hline & Selenophorus sp. 1 & 3 & 1 & 4 \\
\hline & Selenophorus sp. 2 & 4 & & 4 \\
\hline & Stenolophus sp. & 1 & & 1 \\
\hline & Teiresia sp. 1 & 2 & 28 & 30 \\
\hline & Teiresia sp. 2 & 4 & & 4 \\
\hline & Teiresia sp. 3 & 6 & & 6 \\
\hline & Tetragonoderus (Peronoscelis) deuvei Shpeley \& Ball, 2008 & 1 & & 1 \\
\hline Scaritinae & Aspidoglossa agnata Putzeys, 1866 & 3 & & 3 \\
\hline & Semiardistomis sp. & 1 & & 1 \\
\hline Trechinae & Bembidiini sp. & 1 & & 1 \\
\hline CERAMBYCIDAE & & & & \\
\hline Cerambycinae & Achryson surinamum (Linnaeus, 1767) & 7 & & 7 \\
\hline & Aleiphaquilon castaneum (Gounelle, 1911) & 2 & 1 & 3 \\
\hline
\end{tabular}


Tab. II. Cont.

\begin{tabular}{|c|c|c|c|c|}
\hline $\begin{array}{l}\text { FAMÍLIA } \\
\text { Subfamília }\end{array}$ & Espécie/morfoespécie & $\mathrm{MC}$ & VX & Total \\
\hline \multirow{26}{*}{ Lamiinae } & Ambonus electus (Gahan, 1903) & 2 & 3 & 5 \\
\hline & Cupanoscelis heteroclita Gounelle, 1909 & 1 & 3 & 4 \\
\hline & Eburodacrys crassimana Gounelle, 1909 & 8 & 9 & 17 \\
\hline & Isthmiade macilenta Bates, 1873 & 1 & & 1 \\
\hline & Mephritus collidioides (Bates, 1870) & & 2 & 2 \\
\hline & Oxymerus aculeatus Dupont, 1838 & & 1 & 1 \\
\hline & Paranyssicus conspicilattus (Erichson, 1847) & 7 & 11 & 18 \\
\hline & Plocaederus barauna Martins, 2002 & 4 & & 4 \\
\hline & Temnopis megacephala (Germar, 1824) & 3 & & 3 \\
\hline & Antodice kyra Martins \& Galileo, 1998 & 2 & & 2 \\
\hline & Ataxia albisetosa Breuning, 1940 & 1 & & 1 \\
\hline & Baryssinus sp. & & 1 & 1 \\
\hline & Bebelis aff. occulta (Bates, 1866) & 1 & 1 & 2 \\
\hline & Bebelis prolongata (Fisher, 1947) & & 1 & 1 \\
\hline & Bisaltes (Bisaltes) sp. & 1 & 2 & 3 \\
\hline & Dorcasta quadrispinosa Breuning, 1940 & 1 & 1 & 2 \\
\hline & Estola $\mathrm{sp}$. & 9 & 2 & 11 \\
\hline & Exalphus sp. & 5 & 1 & 6 \\
\hline & Hippopsis tuberculata Galileo \& Martins, 1988 & 66 & 10 & 76 \\
\hline & Lepturges (Lepturges) anceps Gilmour, 1962 & 1 & & 1 \\
\hline & Leptostylus perniciosus Monné \& Hoffmann, 1981 & 9 & 7 & 16 \\
\hline & Lypsimena fuscata Haldeman, 1847 & & 1 & 1 \\
\hline & Oreodera aerumnosa Erichson, 1847 & & 1 & 1 \\
\hline & Oreodera glauca glauca (Linnaeus, 1758) & & 1 & 1 \\
\hline & Psapharochrus jaspideus (Germar, 1824) & 4 & 7 & 11 \\
\hline & Pseudomecas pickeli (Melzer, 1930) & 8 & 8 & 16 \\
\hline \multicolumn{5}{|l|}{ CHELONARIIDAE } \\
\hline & Chelonarium sp. & & 5 & 5 \\
\hline \multicolumn{5}{|l|}{ CHRYSOMELIDAE } \\
\hline \multirow[t]{19}{*}{ Bruchinae } & Acanthoscelides sp. 1 & 340 & 24 & 364 \\
\hline & Acanthoscelides sp. 2 & 18 & 1 & 19 \\
\hline & Acanthoscelides sp. 3 & 27 & 1 & 28 \\
\hline & Acanthoscelidina sp. & 1 & & 1 \\
\hline & Amblycerus sp. & 34 & 2 & 36 \\
\hline & Callosobruchus sp. & 1 & & 1 \\
\hline & Caryedes sp. 1 & 34 & 38 & 72 \\
\hline & Caryedes sp. 2 & 1 & & 1 \\
\hline & Megacerus sp. 1 & 26 & & 26 \\
\hline & Megacerus sp. 2 & 1 & & 1 \\
\hline & Megacerus sp. 3 & 1 & & 1 \\
\hline & Megacerus sp. 4 & 1 & & 1 \\
\hline & Meibomeus sp. & 2 & 2 & 4 \\
\hline & Merobruchus columbinus (Sharp, 1885) & 149 & 28 & 177 \\
\hline & Merobruchus sp. & 31 & 40 & 71 \\
\hline & Sennius sp.1 & 5 & & 5 \\
\hline & Sennius sp. 2 & 128 & 8 & 136 \\
\hline & Zabrotes sp. 1 & 62 & 129 & 191 \\
\hline & Zabrotes sp. 2 & 184 & 60 & 244 \\
\hline \multirow{6}{*}{ Cassidinae } & Agroiconota lateripunctata Spaeth, 1936 & 7 & & 7 \\
\hline & Anisostena (Anisostena) sp. & 1 & & 1 \\
\hline & Charidotis sp. 1 & 1 & 1 & 2 \\
\hline & Chalepus notulus (Chapuis, 1877) & 2 & & 2 \\
\hline & Chariodotella (Chariodotella) striatopunctata (Boheman, 1855) & 9 & 15 & 24 \\
\hline & Oxychalepus externus (Chapuis, 1877) & 1 & & 1 \\
\hline \multirow[t]{2}{*}{ Chrysomelinae } & Plagiodera sp. 1 & 28 & 111 & 139 \\
\hline & Plagiodera sp. 2 & & 1 & 1 \\
\hline \multirow[t]{2}{*}{ Criocerinae } & Lema sp. 1 & & 2 & 2 \\
\hline & Lema sp. 2 & & 2 & 2 \\
\hline \multirow[t]{7}{*}{ Cryptocephalinae } & Aratea costata Lacordaire, 1848 & 1 & & 1 \\
\hline & Chlamisus sp. & 1 & & 1 \\
\hline & Cryptocephalus sp. & 1 & 1 & 2 \\
\hline & Pachybrachys sp. 1 & 2 & & 2 \\
\hline & Pachybrachys sp. 2 & 13 & 6 & 19 \\
\hline & Pachybrachys sp. 3 & 31 & 110 & 141 \\
\hline & Pachybrachys sp. 4 & 1 & & 1 \\
\hline
\end{tabular}


Tab. II. Cont.

\begin{tabular}{|c|c|c|c|c|}
\hline $\begin{array}{l}\text { FAMÍLIA } \\
\text { Subfamília }\end{array}$ & Espécie/morfoespécie & $\mathrm{MC}$ & $\mathrm{VX}$ & Total \\
\hline & Temnodachrys (Temnodachrys) signatipennis (Lacordaire, 1848) & 1 & & 1 \\
\hline \multirow[t]{14}{*}{ Eumolpinae } & Colaspis sp. 1 & 5 & 1 & 6 \\
\hline & Colaspis sp. 2 & 3 & & 3 \\
\hline & Colaspis sp. 3 & 1 & 1 & 2 \\
\hline & Colaspis klugii Bowditch, 1921 & 1 & & 1 \\
\hline & Costalimaita ferruginea vulgata (Lefèvre, 1885) & 5 & 1 & 6 \\
\hline & Iphimeis sp. & 2 & 5 & 7 \\
\hline & Megascelis sp. & 1 & 6 & 7 \\
\hline & Myochrous sp. & 3 & & 3 \\
\hline & Paria sp. 1 & 6 & 2 & 8 \\
\hline & Paria sp. 2 & 17 & & 17 \\
\hline & Paria sp. 3 & 1 & & 1 \\
\hline & Rhabdopterus sp. 1 & & 11 & 11 \\
\hline & Rhabdopterus sp. 2 & 12 & 6 & 18 \\
\hline & Typophorus sp. & 1 & & 1 \\
\hline \multirow[t]{26}{*}{ Galerucinae } & Acalymma sp. 1 & & 7 & 7 \\
\hline & Acalymma sp. 2 & 1 & & 1 \\
\hline & Alagoasa sp. & 1 & & 1 \\
\hline & Cerotoma $\mathrm{sp}$. & 4 & & 4 \\
\hline & Chaetocnema sp. & 12 & 1 & 13 \\
\hline & Cornulactica varicornis (Jacoby, 1892) & 1 & & 1 \\
\hline & Cyrcylus sp. 1 & 1 & & 1 \\
\hline & Cyrsylus sp. 2 & 1 & & 1 \\
\hline & Diabrotica sp. & 3 & & 3 \\
\hline & Diabrotica speciosa (Germar, 1824) & 16 & 4 & 20 \\
\hline & Dorisina sp. 1 & 2 & 22 & 24 \\
\hline & Dorisina sp. 2 & & 2 & 2 \\
\hline & Eccoptopsis sp. & 3 & & 3 \\
\hline & Gioia sp. & 1 & & 1 \\
\hline & Hypolampsis sp. & 1 & & 1 \\
\hline & Leptophysa sp. & 2 & 3 & 5 \\
\hline & Longitarsus sp. & 20 & 10 & 30 \\
\hline & Margaridisa $\mathrm{sp}$. & 11 & 14 & 25 \\
\hline & Monomacra sp. 1 & 4 & & 4 \\
\hline & Monomacra sp. 2 & 1 & & 1 \\
\hline & Oedionychus zebratus (Illiger, 1807) & 1 & & 1 \\
\hline & Paralactica $\mathrm{sp}$. & 1 & & 1 \\
\hline & Paranapiacaba sp. & 2 & & 2 \\
\hline & Platyprosopus rubidus Clark, 1865 & 5 & 1 & 6 \\
\hline & Trichaltica $\mathrm{sp.}$ & 2 & & 2 \\
\hline & Wanderbiltiana $\mathrm{sp}$. & & 1 & 1 \\
\hline Sagrinae & Megamerus alvarengai Monrós, 1956 & 0 & 20 & 20 \\
\hline \multicolumn{5}{|l|}{ CLERIDAE } \\
\hline \multirow[t]{2}{*}{ Clerinae } & Axina sp. & 2 & & 2 \\
\hline & Priocera sp. & & 1 & 1 \\
\hline Hydnocerinae & Phyllobaenus sp. & & 3 & 3 \\
\hline \multirow[t]{5}{*}{ Korynetinae } & Corinthiscus sp. 1 & 171 & 20 & 191 \\
\hline & Corinthiscus sp. 2 & 57 & 120 & 177 \\
\hline & Cregya sp. 1 & 1 & 4 & 5 \\
\hline & Cregya sp. 2 & 3 & 4 & 7 \\
\hline & Cregya sp. 3 & 39 & 4 & 43 \\
\hline \multicolumn{5}{|c|}{ COCCINELLIDAE } \\
\hline \multirow[t]{13}{*}{ Coccinellinae } & Cycloneda conjugata (Mulsant, 1850) & 6 & 7 & 13 \\
\hline & Cycloneda sanguinea (L., 1763) & 3 & & 3 \\
\hline & Diomus sp. 1 & 1 & 1 & 2 \\
\hline & Diomus sp. 2 & 97 & 21 & 118 \\
\hline & Gordonoryssomus delicatus Almeida \& Loima, 1995 & 19 & 6 & 25 \\
\hline & Harpasus sp. & 2 & & 2 \\
\hline & Hippodamia convergens Guérin-Méneville, 1842 & 1 & & 1 \\
\hline & Hyperaspis sp. & 15 & 8 & 23 \\
\hline & Psyllobora confluens (Fabricius, 1801) & 39 & 3 & 42 \\
\hline & Stethorus tridens Gordon, 1982 & 3 & & 3 \\
\hline & Tenuisvalvae notata (Mulsant, 1850) & 14 & 1 & 15 \\
\hline & Zenoria luciae González \& Honour, 2012 & 2 & & 2 \\
\hline & Zogloba beaumonti Casey, 1899 & 108 & 12 & 120 \\
\hline
\end{tabular}


Tab. II. Cont.

\begin{tabular}{|c|c|c|c|c|}
\hline $\begin{array}{l}\text { FAMÍLIA } \\
\text { Subfamília }\end{array}$ & Espécie/morfoespécie & $\mathrm{MC}$ & $\mathrm{VX}$ & Total \\
\hline Microweiseinae & Coccidophilus sp. & 3 & 4 & 7 \\
\hline \multicolumn{5}{|l|}{ CORYLOPHIDAE } \\
\hline \multirow[t]{2}{*}{ Corylophinae } & Clypastraea sp. & & 2 & 2 \\
\hline & Sericoderus sp. & 1 & & 1 \\
\hline \multicolumn{5}{|l|}{ CURCULIONIDAE } \\
\hline \multirow[t]{4}{*}{ Baridinae } & Baridinae sp. 1 & 1 & & 1 \\
\hline & Madarina sp. 1 & 6 & 1 & 7 \\
\hline & Parasaldius sp. & 1 & & 1 \\
\hline & Thaliabaridina sp. & 3 & & 3 \\
\hline Ceutorhynchinae & Hypocoeliodes sp. & & 3 & 3 \\
\hline \multirow[t]{3}{*}{ Conoderinae } & Eulechriops sp. 1 & & 2 & 2 \\
\hline & Eulechriops sp. 2 & 11 & 11 & 22 \\
\hline & Lechriops sp. & 2 & 29 & 31 \\
\hline \multirow{9}{*}{ Cryptorhynchinae } & Coelosterninus longipennis (Boheman, 1837) & 4 & & 4 \\
\hline & Coelosternus sp. & 1 & & 1 \\
\hline & Cryptorhynchus sp. 1 & 1 & & 1 \\
\hline & Cryptorhynchus sp. 2 & 1 & & 1 \\
\hline & Eubulus sp. 1 & 5 & 1 & 6 \\
\hline & Eubulus sp. 2 & 1 & & 1 \\
\hline & Malacobius sp. & & 2 & 2 \\
\hline & Pappista sp. & 1 & & 1 \\
\hline & Tyloderma sp. & & 1 & 1 \\
\hline \multirow[t]{4}{*}{ Curculioninae } & Erodiscus caruaru Vanin, 1986 & 1 & & 1 \\
\hline & Sibinia sp. 1 & 23 & 18 & 41 \\
\hline & Sibinia sp. 2 & 2 & & 2 \\
\hline & Sibinia sp. 3 & 34 & 13 & 47 \\
\hline \multirow[t]{10}{*}{ Entiminae } & Cyphopsis clathrata Roelofs, 1879 & & 4 & 4 \\
\hline & Entimini sp. & 4 & 2 & 6 \\
\hline & Lordopini sp. & 1 & & 1 \\
\hline & Naupactus sp. 1 & 2 & 3 & 5 \\
\hline & Naupactus sp. 2 & & 1 & 1 \\
\hline & Pandeleteius sp. & 15 & 28 & 43 \\
\hline & Promecops sp. 1 & 6 & & 6 \\
\hline & Promecops sp. 2 & 16 & 3 & 19 \\
\hline & Promecops sp. 3 & 2 & 16 & 18 \\
\hline & Promecops sp. 4 & 1 & & 1 \\
\hline Hyperinae & Phelypera schuppeli (Boheman, 1834) & & 1 & 1 \\
\hline \multirow[t]{18}{*}{ Molytinae } & Chalcodermus sp. 1 & 8 & & 8 \\
\hline & Chalcodermus sp. 2 & 36 & 7 & 43 \\
\hline & Chalcodermus sp. 3 & 13 & 4 & 17 \\
\hline & Chalcodermus sp. 4 & 1 & & 1 \\
\hline & Chalcodermus sp. 5 & 1 & 1 & 2 \\
\hline & Conotrachelus sp. 1 & 9 & & 9 \\
\hline & Conotrachelus sp. 2 & 7 & & 7 \\
\hline & Conotrachelus sp. 3 & 10 & 1 & 11 \\
\hline & Conotrachelus sp. 4 & 1 & & 1 \\
\hline & Conotrachelus sloaneae Marshall, 1940 & 3 & & 3 \\
\hline & Heilus sp. 1 & 2 & 1 & 3 \\
\hline & Heilus sp. 2 & 2 & & 2 \\
\hline & Pheloconus sp. & 11 & & 11 \\
\hline & Rhyssomatus sp. 1 & 11 & 2 & 13 \\
\hline & Rhyssomatus sp. 2 & 3 & & 3 \\
\hline & Rhyssomatus sp. 3 & & 1 & 1 \\
\hline & Rhyssomatus sp. 4 & 1 & 1 & 2 \\
\hline & Sternechus sp. & & 2 & 2 \\
\hline \multirow[t]{2}{*}{ Platypodinae } & Platypus sp. 1 & 26 & 34 & 60 \\
\hline & Platypus sp. 2 & 9 & 16 & 25 \\
\hline \multirow[t]{5}{*}{ Scolytinae } & Ambrosiodmus sp. & 7 & 2 & 9 \\
\hline & Hypothenemus sp. & 4 & & 4 \\
\hline & Pityophthorus sp. & 1 & & 1 \\
\hline & Xyleborus sp. & 8 & 1 & 9 \\
\hline & Curculionidae sp. 1 & 8 & 2 & 10 \\
\hline \multicolumn{5}{|l|}{ DERMESTIDAE } \\
\hline \multirow[t]{2}{*}{ Megatominae } & Cryptorhopalum sp. & 9 & 3 & 12 \\
\hline & Trogoderma sp. & 2 & 2 & 4 \\
\hline
\end{tabular}


Tab. II. Cont.

\begin{tabular}{|c|c|c|c|c|}
\hline $\begin{array}{l}\text { FAMÍLIA } \\
\text { Subfamília }\end{array}$ & Espécie/morfoespécie & $\mathrm{MC}$ & $\mathrm{VX}$ & Total \\
\hline \multicolumn{5}{|l|}{ DYTISCIDAE } \\
\hline & Dytiscidae sp. & & 1 & 1 \\
\hline \multicolumn{5}{|l|}{ ENDOMYCHIDAE } \\
\hline Stenotarsinae & Stenotarsus sp. & & 1 & 1 \\
\hline \multicolumn{5}{|l|}{ ELATERIDAE } \\
\hline \multirow[t]{11}{*}{ Agrypninae } & Aeolus sp. 1 & 23 & 6 & 29 \\
\hline & Aeolus sp. 2 & 5 & & 5 \\
\hline & Aeolus sp. 3 & 15 & 7 & 22 \\
\hline & Aeolus sp. 4 & 1 & 2 & 3 \\
\hline & Conoderus sp. 1 & 3 & 2 & 5 \\
\hline & Conoderus sp. 2 & 2 & 2 & 4 \\
\hline & Conoderus sp. 3 & 5 & & 5 \\
\hline & Heteroderes sp. 1 & 1 & 3 & 4 \\
\hline & Heteroderes sp. 2 & 4 & & 4 \\
\hline & Pyrearinus sp. 1 & 4 & 13 & 17 \\
\hline & Pyrearinus sp. 2 & & 1 & 1 \\
\hline \multirow[t]{10}{*}{ Cardiophorinae } & Cardiophorus sp. & 4 & 1 & 5 \\
\hline & Esthesopus sp. & 1 & & 1 \\
\hline & Horistonotus sp. 1 & 18 & 3 & 21 \\
\hline & Horistonotus sp. 2 & 121 & 5 & 126 \\
\hline & Horistonotus sp. 3 & 70 & 6 & 76 \\
\hline & Horistonotus sp. 4 & 5 & & 5 \\
\hline & Horistonotus sp. 5 & 8 & 1 & 9 \\
\hline & Horistonotus sp. 6 & 1 & & 1 \\
\hline & Horistonotus sp. 7 & 2 & & 2 \\
\hline & Cardiophorinae sp. & 1 & & 1 \\
\hline \multirow[t]{2}{*}{ Dendrometrinae } & Crepidius sp. 1 & 13 & 15 & 28 \\
\hline & Crepidius sp. 2 & 5 & 1 & 6 \\
\hline \multirow[t]{4}{*}{ Elaterinae } & Anchastus sp. & 4 & & 4 \\
\hline & Agriotes sp. & 6 & 11 & 17 \\
\hline & Cosmesus sp. 1 & 30 & 1 & 31 \\
\hline & Cosmesus sp. 2 & & 1 & 1 \\
\hline \multicolumn{5}{|l|}{ HISTERIDAE } \\
\hline Histerinae & Exosternini sp. 1 & 1 & & 1 \\
\hline \multicolumn{5}{|l|}{ LAMPYRIDAE } \\
\hline \multirow[t]{4}{*}{ Lampyrinae } & Aspisoma sp. 1 & 1 & & 1 \\
\hline & Aspisoma sp. 2 & 2 & & 2 \\
\hline & Cratomorphini sp. 1 & 1 & & 1 \\
\hline & Photinus sp. & 93 & 3 & 96 \\
\hline \multicolumn{5}{|l|}{ LATRIDIIDAE } \\
\hline Corticariinae & Melanophthalma sp. & 5 & 1 & 6 \\
\hline \multicolumn{5}{|l|}{ LEIODIDAE } \\
\hline Leiodinae & Cyrtusa sp. & 5 & 2 & 7 \\
\hline \multicolumn{5}{|l|}{ MELOIDAE } \\
\hline \multirow[t]{3}{*}{ Meloinae } & Epicauta grammica (Fischer, 1827) & 3 & & 3 \\
\hline & Epicauta sp. 1 & 1 & & 1 \\
\hline & Pyrota vittigera Blanchard, 1843 & & 1 & 1 \\
\hline Eleticinae & Spastica $\mathrm{sp}$. & & 1 & 1 \\
\hline MELYRIDAE & & & & \\
\hline Malachiinae & Attalus sp. & 1 & 2 & 3 \\
\hline MORDELLIDAE & & & & \\
\hline Mordellinae & Mordella sp. 1 & 10 & 36 & 46 \\
\hline & Mordella sp. 2 & 2 & 11 & 13 \\
\hline & Mordella sp. 4 & & 7 & 7 \\
\hline & Mordella sp. 5 & 10 & 80 & 90 \\
\hline & Mordella sp. 6 & 4 & 16 & 20 \\
\hline & Mordella sp. 7 & & 1 & 1 \\
\hline & Mordellistena sp. 1 & 28 & 41 & 69 \\
\hline & Mordellistena sp. 2 & 92 & 55 & 147 \\
\hline & Mordellistena sp. 3 & 50 & 34 & 84 \\
\hline & Mordellistena sp. 4 & 2 & 3 & 5 \\
\hline & Mordellistena sp. 5 & 2 & & 2 \\
\hline NITIDULIDAE & & & & \\
\hline Carpophilinae & Carpophilus (Carpophilus) hemipterus (Linnaeus, 1758) & 3 & & 3 \\
\hline & Carpophilus (Megacarpolus) lugubris Murray, 1864 & 2 & & 2 \\
\hline
\end{tabular}


Tab. II. Cont.

\begin{tabular}{|c|c|c|c|c|}
\hline $\begin{array}{l}\text { FAMÍLIA } \\
\text { Subfamília }\end{array}$ & Espécie/morfoespécie & $\mathrm{MC}$ & VX & Total \\
\hline & Carpophilus (Myothorax) dimidiatus (Fabricius, 1792) & 1 & 1 & 2 \\
\hline Cybocephalinae & Cybocephalus sp. & 1 & 1 & 2 \\
\hline \multirow[t]{2}{*}{ Cyllaeinae } & Colopterus sp. & 12 & 3 & 15 \\
\hline & Colopterus vulneratus (Erichson, 1843) & 1 & & 1 \\
\hline \multirow[t]{3}{*}{ Nitidulinae } & Lobiopa sp. & 5 & 1 & 6 \\
\hline & Mystrops sp. & 1 & 1 & 2 \\
\hline & Stelidota $\mathrm{sp}$ & 7 & 2 & 9 \\
\hline \multicolumn{5}{|l|}{ OEDEMERIDAE } \\
\hline Oedemerinae & Oxacis sp. & 1 & 1 & 2 \\
\hline Polypriinae & Polypria sp. & 10 & 15 & 25 \\
\hline \multicolumn{5}{|l|}{ PHALACRIDAE } \\
\hline \multirow[t]{2}{*}{ Phalacrinae } & Acylomus sp. & 153 & 66 & 219 \\
\hline & Stilbus sp. & 30 & 5 & 35 \\
\hline \multicolumn{5}{|l|}{ PHENGODIDAE } \\
\hline \multirow[t]{3}{*}{ Mastinocerinae } & Nephroma sp. & 17 & 3 & 20 \\
\hline & Stenophrixothrix sp. & 14 & 54 & 68 \\
\hline & Taximastinocerus sp. & 5 & 6 & 11 \\
\hline Phengodinae & Phengodes sp. & 6 & 3 & 9 \\
\hline \multicolumn{5}{|l|}{ PTILODACTYLIDAE } \\
\hline Ptilodactylinae & Ptilodactyla sp. & 7 & & 7 \\
\hline \multicolumn{5}{|l|}{ PTINIDAE } \\
\hline Anobiinae & Petalius sp. & 1 & 1 & 2 \\
\hline \multirow[t]{2}{*}{ Dorcatominae } & Caenocara sp. 1 & 7 & 11 & 18 \\
\hline & Caenocera sp. 2 & & 1 & 1 \\
\hline \multirow[t]{2}{*}{ Mesocoelopodinae } & Tricorynus sp. 1 & 78 & 12 & 90 \\
\hline & Tricorynus sp. 2 & 1 & & 1 \\
\hline \multirow[t]{3}{*}{ Ptininae } & Lasioderma serricornis (Fabricius, 1792) & 2 & 2 & 4 \\
\hline & Ptinus sp. 1 & 21 & 1 & 22 \\
\hline & Ptinus sp. 2 & & 1 & 1 \\
\hline \multicolumn{5}{|l|}{ SCARABAEIDAE } \\
\hline Aphodiinae & Ataenius sp. 1 & 5 & 2 & 7 \\
\hline Bolboceratinae & Bolbapium sp. 1 & 1 & 17 & 18 \\
\hline \multirow[t]{2}{*}{ Rutelinae } & Leucothyreus $\mathrm{sp} .1$ & & 2 & 2 \\
\hline & Lobogeniates sp. 1 & 1 & & 1 \\
\hline Scarabaeinae & Digitonthophagus gazella (Fabricius, 1787) & 1 & & 1 \\
\hline & Deltochilum verruciferum Felsche, 1911 & & 1 & 1 \\
\hline & Onthophagus sp. & 1 & & 1 \\
\hline Melolonthinae & Clavipalpus dejeanii Laporte, 1832 & & 1 & 1 \\
\hline & Liogenys sp. & 1 & & 1 \\
\hline SCRAPTIIDAE & & & & \\
\hline Scraptiinae & Scraptia sp. & 8 & & 8 \\
\hline SCIRTIDAE & & & & \\
\hline Scirtinae & Ora sp. & 29 & 1 & 30 \\
\hline SILVANIDAE & & & & \\
\hline Silvaninae & Ahasverus advena Waltl, 1832 & 2 & & 2 \\
\hline & Silvaninae sp. 1 & 1 & & 1 \\
\hline STAPHYLINIDAE & & & & \\
\hline Aleocharinae & Aleocharinae sp. 1 & & 1 & 1 \\
\hline & Aleocharinae sp. 2 & 2 & & 2 \\
\hline & Aleocharinae sp. 3 & 2 & & 2 \\
\hline & Aleocharinae sp. 4 & 1 & & 1 \\
\hline Megalopsidiinae & Megalopinus sp. & & 4 & 4 \\
\hline Paederinae & Palaminus sp. & 1 & & 1 \\
\hline & Paederinae sp. 1 & 1 & & 1 \\
\hline & Paederinae sp. 2 & 1 & & 1 \\
\hline & Paederinae sp. 3 & 1 & & 1 \\
\hline & Paederinae sp. 4 & 4 & & 4 \\
\hline & Paederinae sp. 5 & 1 & & 1 \\
\hline & Paederinae sp. 6 & & 1 & 1 \\
\hline & Taenoderma sp. & & 1 & 1 \\
\hline Tachyporinae & Coproporus sp. & 20 & & 20 \\
\hline Scaphidiinae & Baeocera sp. & & 1 & 1 \\
\hline Scydmaeninae & Euconnus sp. & 2 & & 2 \\
\hline Staphylininae & Acylophorus sp. 1 & 1 & & 1 \\
\hline & Acylophorus sp. 2 & 1 & & 1 \\
\hline
\end{tabular}


Tab. II. Cont.

\begin{tabular}{|c|c|c|c|c|}
\hline $\begin{array}{l}\text { FAMÍLIA } \\
\text { Subfamília }\end{array}$ & Espécie/morfoespécie & $\mathrm{MC}$ & $\mathrm{VX}$ & Total \\
\hline & Belonuchus sp. & 4 & & 4 \\
\hline & Diochus sp. 1 & 2 & & 2 \\
\hline & Diochus sp. 2 & 12 & & 12 \\
\hline & Philonthus sp. 2 & 1 & & 1 \\
\hline & Philonthus sp. 3 & & 1 & 1 \\
\hline & Xantholinini sp. 1 & 1 & & 1 \\
\hline & Xantholinini sp. 2 & 1 & & 1 \\
\hline & Xantholinini sp. 3 & 1 & & 1 \\
\hline \multicolumn{5}{|l|}{ TENEBRIONIDAE } \\
\hline \multirow[t]{4}{*}{ Alleculinae } & Allecula sp. 1 & 41 & 5 & 46 \\
\hline & Allecula sp. 2 & 1 & & 1 \\
\hline & Cteisa sp. & 2 & & 2 \\
\hline & Lobopoda sp. & & 1 & 1 \\
\hline Diaperinae & Poecilocrypticini formicophilus Gebien, 1928 & 2 & & 2 \\
\hline \multirow[t]{6}{*}{ Lagriinae } & Anaedus sp. & 16 & & 16 \\
\hline & Epitragus (Epitragus) sp. & 4 & 5 & 9 \\
\hline & Epitragus (Similepitragus) sp. 1 & 59 & 4 & 63 \\
\hline & Epitragus (Similepitragus) sp. 2 & 277 & 53 & 330 \\
\hline & Lagria villosa (Fabricius, 1783) & 21 & & 21 \\
\hline & Statira sp. & 9 & & 9 \\
\hline Pimeliinae & Epitragus sp. & 2 & & 2 \\
\hline \multirow[t]{3}{*}{ Stenochinae } & Opatrini sp. 1 & 1 & & 1 \\
\hline & Talanini sp. & 1 & & 1 \\
\hline & Tenebrionidae sp. 1 & 1 & & 1 \\
\hline THROSCIDAE & Aulonothroscus sp. & 17 & & 17 \\
\hline \multicolumn{5}{|l|}{ TROGOSSITIDAE } \\
\hline \multirow[t]{3}{*}{ Trogossitinae } & Tenebroides sp. 1 & 2 & 1 & 3 \\
\hline & Tenebroides sp. 2 & 2 & & 2 \\
\hline & Tenebroides sp. 3 & 2 & 1 & 3 \\
\hline \multicolumn{5}{|l|}{ ZOPHERIDAE } \\
\hline \multirow[t]{3}{*}{ Colidiinae } & Bitoma sp. & 1 & & 1 \\
\hline & Pharax luticollis Pascoe, 1860 & 1 & & 1 \\
\hline & Sinchitini sp. & 23 & 1 & 24 \\
\hline \multirow[t]{2}{*}{ Zopherinae } & Hyporhagus sp. 1 & & 8 & 8 \\
\hline & Hyporhagus sp. 2 & 47 & 56 & 103 \\
\hline
\end{tabular}

\section{DISCUSSÃO}

Considerando que se trata de uma amostragem com apenas um tipo de armadilha, o esforço amostral resultou em relevante representatividade da fauna de besouros da região, equivalente a $37,7 \%$ do total de famílias de Coleoptera registradas para o Brasil (Monné \& Costa, 2018). Entretanto, ressalta-se que, como se trata de uma armadilha de interceptação, com captura na parte superior da mesma, a amostragem se restringiu àquelas famílias que possuem hábitos de desviar de obstáculos voando mais para cima. Evidencia-se ainda que a amostragem de alguns táxons específicos de Coleoptera apresenta restrições pela falta de métodos variados, iscas atrativas e, por isso, muitas vezes acabam não sendo capturados ou raramente coletados.

Deve-se considerar que, em geral, a composição de famílias registradas nesse estudo foi consequência do método de coleta utilizado. A armadilha Malaise propicia a captura de espécies de besouros com maior capacidade de deslocamento e de tamanho reduzido, incluindo as que se movimentam entre as áreas, ou seja, captura possivelmente indivíduos de espécies mais transitórias que outros métodos (GANHO \& MARINONI, 2006), como por exemplo, as armadilhas de solo. O número e a composição de famílias registradas é similar ao observado em nove fitofisionomias de Caatinga Meridional, amostradas em Alagoas e Sergipe por IANNUZZI et al. (2003).

As famílias registradas que apresentaram as maiores riquezas (e.g., Chrysomelidae, Curculionidae, Cerambycidae, Carabidae, Staphylinidae) possuem elevada diversidade (BoUCHARD et al., 2017) e são funcionalmente importantes nos ecossistemas ao desempenharem atividades como a herbivoria - algumas vindo a ser pragas -, a degradação de madeira e a predação de outros insetos (MARINONI et al., 2001; CASARI \& IDE, 2012).

Em relação às famílias mais abundantes, IANNUZZI et al. (2003) obtiveram resultados similares em estudos envolvendo diferentes fitofisionomias de Caatinga Meridional em Alagoas e Sergipe, sendo Elateridae, Chrysomelidae, Mordellidae, Tenebrionidae e Curculionidae as mais representativas, respectivamente. MARINONI \& DUTRA (1997) e GANHO \& MARINONI (2006) também registraram Chrysomelidae, Curculionidae, Mordellidae e Elateridae entre as mais abundantes em levantamentos no Paraná. Em fragmento de Mata Atlântica, no Rio Grande do Norte, LIMA et al. (2010) verificaram Chrysomelidae, Coccinellidae, Mordellidae, Curculionidae e Elateridae como as mais representativas. Isso demonstra que a Malaise é uma 
armadilha eficiente, podendo ser recomendada para a captura dessas famílias de Coleoptera.

Das famílias que mais se destacaram por sua diversidade nesse estudo, têm-se: Chrysomelidae, que entre os Coleoptera sobressai como a quarta maior família em número de espécies, sendo um grupo predominantemente fitófago, utilizando todas as partes da planta como alimento dependendo da subfamília (MARINONI et al., 2001; CASARI \& IDE, 2012; BouchaRD et al., 2017). Chrysomelidae apresenta importância ecológica e econômica, com algumas espécies causando prejuízos em culturas importantes, enquanto outras são usadas eficientemente no controle de ervas daninhas (ForTi et al., 2011; CASARI \& IDE, 2012). Neste estudo, foram amostradas as nove subfamílias de Chrysomelidae de ocorrência no Brasil, do total de 12 que compõem a família (BOUCHARD et al., 2011). Os representantes de Bruchinae, principal subfamília entre os crisomelídeos amostrados neste estudo, são registrados em 36 famílias de plantas hospedeiras (RIBEIRO-Costa \& AlmeIDA, 2012) das quais 70\% pertencem à Fabaceae (Morse, 2014). Esse fato justifica a grande representatividade de Bruchinae, considerando toda a área estudada, uma vez que a elevada ocorrência de Fabaceae na mesma é evidente. Acrescenta-se ainda que é a família botânica com maior riqueza na Caatinga (BFG, 2015).

Dentre os crisomelídeos identificados, Megamerus alvarengai Monrós, 1956 (MonRós, 1956) merece atenção, pois o único registro existente até então era o da descrição original da mesma; a série-tipo foi coletada em Natal (RN) na década de 1950. A espécie é a única representante no Brasil de Sagrinae e esse é o primeiro registro para o estado da Paraíba. $\mathrm{O}$ fato da coleta dessa espécie reforça a necessidade de conservação dos remanescentes de Caatinga da região estudada, bem como, torna o conhecimento gerado a partir desta pesquisa, de maior importância por se tratar de uma região com pouco conhecimento sobre Coleoptera do bioma em questão. Justifica-se tal estratégia, pois a área de estudo está localizada em uma das áreas indicadas como prioritárias para a conservação da biodiversidade da Caatinga, sendo classificada como de alta importância biológica (Patos/Santa Terezinha) (SILVA et al., 2003) e próxima à área categorizada como de muito alta importância biológica (Seridó/Serra de Santa Luzia) (BRANDÃo et al., 2003), o que aumenta a necessidade de conservação dessa vegetação.

Curculionidae, a segunda família mais rica em espécies neste estudo, é considerada um dos grupos mais bem-sucedidos dentre os Coleoptera, com grande variedade de hábitos alimentares e ampla distribuição geográfica (LAWRENCE \& BRITTON, 1991), além de conter o segundo maior número de espécies de Coleoptera, sendo essencialmente herbívora (MARINONI et al., 2001; BOUCHARD et al., 2017). A maior parte dos adultos são estritamente fitófagos, e as larvas apresentam, em geral, estreita variedade de plantas hospedeiras (CASARI \& IDE, 2012). O fato de serem insetos principalmente herbívoros, provavelmente, favorece a família a ser uma das mais diversas e abundantes em vários levantamentos de besouros (GANHO \& MARINONI, 2003; IANNUZZI et al., 2003; GANHO \& MARINONI, 2006; AuAD \& CARVALHO, 2011).
Destacou-se também Cerambycidae dentre as famílias mais diversas nesse estudo. Das espécies coletadas, a maior parte foi reportada por NASCIMENTO et al. (2017) que reúnem informações de diversos cerambicídeos ocorrentes no semiárido brasileiro; no entanto, 11 delas não estão na lista organizada por esses autores. Além disso, cinco desses táxons [Isthmiade macilenta Bates, 1873, Dorcasta quadrispinosa Breuning, 1940, Bebelis prolongata (Fisher, 1947), Hippopsis tuberculata Galileo \& Martins, 1988 e Antodice kyra Martins \& Galileo, 1998], além de Aleiphaquilon castaneum (Gounelle, 1911), são novos registros para o estado da Paraíba.

A quantidade de espécies com um único indivíduo na comunidade amostrada segue o padrão observado em outros estudos. GANHO \& MARINONI (2006) registraram resultados semelhantes aos deste estudo, com um elevado número de espécies singletons para Coleoptera no Paraná; padrão similar foi verificado por LINZMEIER et al. (2006) para Alticini (Coleoptera) no mesmo estado. A quantidade de singletons foi um pouco maior do que a expectativa de $32 \%$ relatada por CoDDINGTON et al. (2009) em inventários de comunidades tropicais. Apesar, que, de acordo com esses autores, a provável causa para a elevada percentagem de espécies raras em ecossistemas tropicais é a subamostragem, que determina uma tendência positiva nas estimativas de riqueza real de espécies. Os mesmos autores propõem reduzir o efeito da subamostragem pela simples ampliação do esforço, de forma a acrescentar mais indivíduos por espécies no inventário. No entanto, pode-se observar que para a região estudada, mesmo com um levantamento longo de quatro anos, o percentual não foi reduzido, sendo maior do que o percentual de expectativa. Isso pode ser um indício que as espécies singletons e doubletons obtidas são comuns em coletas com Malaise na Caatinga e, indica ainda que deve haver um elevado número de espécies a ser registrado na área estudada. Assim, o uso de outros métodos de amostragem poderá fazer com que o número de singletons e de doubletons seja reduzido.

As espécies mais abundantes foram representantes de quatro famílias, a maior parte delas com hábito alimentar fitófago. Possivelmente, a diversidade de famílias registradas dentre as espécies mais abundantes foi favorecida devido à amostragem ter incluído mais de uma fitofisionomia de Caatinga, uma vez que, teve-se o propósito de verificar a efetividade de coleta de besouros com armadilhas Malaise nesse bioma.

Com relação ao esforço de amostragem, a curva de acumulação de espécies não atingiu uma assíntota, indicando que, apesar de longo, o período amostral ainda não foi suficiente para que houvesse estabilização da curva. Esse problema é intensificado quando se pesquisam invertebrados, especialmente para grupos hiperdiversos, porém, mal conhecidos, como artrópodes (Colwell \& Coddington, 1994; Jiménez-VALVERde \& Hortal, 2003). Além disso, o tipo de armadilha utilizada e o elevado número de singletons e doubletons registrado foram determinantes para esse resultado.

A diversidade de besouros aqui apresentada amplia o conhecimento de Coleoptera no Nordeste brasileiro e 
soma-se a outros trabalhos encontrados na literatura que trazem inventários conduzidos na Caatinga. Dentre estes, destacam-se os estudos com besouros necrófagos em carcaças na Bahia (SAntos \& SAntos, 2016), na Paraíba (SAntos et al., 2014) e em Pernambuco (MAYER \& VASCONCELOS, 2013).

Os resultados obtidos revelam um número considerável de espécies de besouros no bioma Caatinga. O fato de se ter tido registros relevantes, a exemplo da $M$. alvarengai (Chrysomelidae: Sagrinae) como o primeiro para a espécie após 60 anos da sua descrição, demonstra a importância da conservação dos remanescentes de Caatinga estudados para a manutenção da coleopterofauna dessa região, bem como de seus serviços ecossistêmicos. A posterior análise dos dados por fitofisionomia proporcionará mais inferências para a fauna de besouros na região estudada. Sugere-se, complementar o inventário com a utilização de outros métodos de amostragens, o que irá permitir a coleta de espécies que utilizam outros hábitos de dispersão e que não são amostrados com o uso de armadilhas Malaise.

Agradecimentos. Ao Sr. Pierre Lantolt proprietário da Fazenda Tamanduá pela permissão para as coletas, ao $\mathrm{CNPq}$, por ter financiado o projeto de pesquisa "Diversidade, Ecologia e Conservação de Himenópteros na Região Semiárido do Nordeste do Brasil, com Ênfase nas Abelhas", em nome de FCVZ, o que permitiu a realização das amostragens, ao Sr. Ayr de Moura Bello (Rio de Janeiro-RJ), a Profa. Dra. Lúcia Massuti Almeida (Universidade Federal do Paraná, Curitiba-PR) e ao Dr. Angelico Fortunato Asenjo Flores (Universidade Federal do Mato Grosso, Cuiabá-MT), pelas identificações dos besouros.

\section{REFERÊNCIAS BIBLIOGRÁFICAS}

Álvares, C. A.; Stape, J. L.; Sentelhas, P. C.; GonÇalves, J. L. M. \& SparoveK, G. 2013. Köppen's climate classification map for Brazil. Meteorologische Zeitschrift 22(6):711-728.

Arnett JR., R. H. \& Thomas, M. C. 2001. American beetles. Vol. 1: Archostemata, Myxophaga, Adephaga, Polyphaga: Staphyliniformia. Boca Raton, CRC Press. 443p.

Arnett JR., R. H.; Thomas, M. C.; Skelley, P. E. \& Frank, J. H. 2002. American beetles. Vol. 2: Polyphaga: Scarabaeoidea through Curculionoidea. Boca Raton, CRC Press. 861p.

Aund, A. M. \& CARvalho, C. A. 2011. Análise faunística de Coleópteros em sistema silvipastoril. Ciências Florestais 21(1):31-39.

BFG. 2015. Growing knowledge: an overview of Seed Plant diversity in Brazil. Rodriguésia 66(4):1085-1113.

Воотн, R. G.; Cox, M. L. \& MADGE, R. B. 1990. IIE guides to insects of importance to man. 3. Coleoptera. Wallingford, CAB International. 384p.

Bouchard, P. 2014. The book of Beetles. A life size guide to six hundred of nature's gems. London, Ivy Press Limited. 656p.

Bouchard, P.; Bousquet, Y.; Davies, A. E.; Alonso-Zarazaga, M. A.; Lawrence, J. F.; Lyal, C. H. C.; Newton, A. F.; Reid, C. A. M.; SchmitT, M.; Ślipiński, S. A. \& Smith, A. B. T. 2011. Family-group names in Coleoptera (Insecta). ZooKeys 88:1-972.

Bouchard, P.; Smith, A. B. T.; Douglas, H.; Gimmel, M. L.; Brunke, A. J. \& KANDA, K. 2017. Biodiversity of Coleoptera. In: FootTIT, R. G. \& ADLER, P. H. eds. Insect Biodiversity: science and society. 2 edn. Hoboken, John Wiley \& Sons, p. 337-417.

Brandẽo, C. R. F.; Viana, B. F.; Martins, C. F.; Yamamoto, C. I.; Zanella, F. C. V. \& CASTRO, M. 2003. Invertebrados: áreas e ações prioritárias para a conservação da Caatinga. In: Silva, J. M. C.; TABarelli, M.; Fonseca, M. T. \& Lins, L. V. eds. Biodiversidade da Caatinga: áreas e ações prioritárias para a conservação. Brasília, Ministério do Meio Ambiente, p. 141-147.

BRANDÃo, C. R. F. \& YAмAmoto, C. I. 2003. Invertebrados da Caatinga. In: Silva, J. M. C.; Tabarelli, M.; Fonseca, M. T. \& Lins, L. V. eds. Biodiversidade da Caatinga: áreas e ações prioritárias para a conservação. Brasília, Ministério do Meio Ambiente, p. 136-140.
Calor, A. R. \& Bravo, F. 2014. Artrópodes do semiárido: conhecimento atual e desafios para os próximos anos. In: Bravo, F. \& CALOR, A. R. eds. Artrópodes do semiárido: biodiversidade e conservação. Feira de Santana, Printmídia, p. 293-296.

CASARI, S. A. \& IDE, S. 2012. Coleoptera Linnaeus, 1758. In: Rafael, J. A.; Melo, G. A. R.; Carvalho, C. J. B.; Casari, S. A. \& Constantino, R. Insetos do Brasil: diversidade e taxonomia. Ribeirão Preto, Ed. Holos, p. 454-535.

Castelletti, C. H. M.; Silva, J. M. C.; Tabarelli, M. \& Santos, A. M. M. 2003. Quanto ainda resta da Caatinga? Uma estimativa preliminar. In: Silva, J. M.; Tabarelli, M.; Fonseca, M. T. \& Lins, L. V. eds. Biodiversidade da Caatinga: áreas e ações prioritárias para a conservação. Brasília, Ministério do Meio Ambiente/Universidade Federal de Pernambuco, p. 91-100.

Coddington, J. A.; Agnarsson, I.; Miller, J. A.; Kuntner, M. \& Hormiga, G. 2009. Undersampling bias: the null hypothesis for singleton species in tropical arthropod surveys. Journal of Animal Ecology 78(3):573-584.

Colwell, R. K. \& Coddington, J. A. 1994. Estimating terrestrial biodiversity through extrapolation. Philosophical Transactions of the Royal Society B Biological Sciences 345:101-118.

Constantino, R.; Diniz, I. R.; Pujol-Luz, J. R.; Motta, P. C. \& Laumann, R. A. 2002. Textos de Entomologia, versão 3. Brasília, Universidade de Brasília. 89p.

Endres, A. A.; Hernández M. I. M. \& Creão-Duarte. A. J. 2005. Considerações sobre Coprophanaeus ensifer (Germar) (Coleoptera, Scarabaeidae) em um remanescente de Mata Atlântica no Estado da Paraíba, Brasil. Revista Brasileira de Entomologia 49(3):427-429.

Endres, A. A.; Creão-Duarte, A. J. \& Hernández, M. I. M. 2007. Diversidade de Scarabaeidae s. str. (Coleoptera) da Reserva Biológica Guaribas, Mamanguape, Paraíba, Brasil: uma comparação entre Mata Atlântica e Tabuleiro Nordestino. Revista Brasileira de Entomologia 51(1):67-71.

Forti, L. C.; Moreira, S. M.; Caldato, N. \& Baldin, E. L. L. 2011. Ordem Coleoptera. In: FuJihara, R. T.; Forti, L. C.; Almeida, M. C. \& Baldin, E. L. L. eds. Insetos de importância econômica: guia ilustrado para identificação de famílias. Botucatu, Editora FEPAF, p. 187-225.

Ganho, N. G. \& Marinoni, R. C. 2003. Fauna de Coleoptera no Parque Estadual de Vila Velha, Ponta Grossa, Paraná, Brasil. Abundância e riqueza das famílias capturadas através de armadilhas malaise. Revista Brasileira de Zoologia 20(4):727-736.

GANHO, N. G. \& MARINONI, R. C. 2006. Variabilidade espacial das famílias de Coleoptera (Insecta) entre fragmentos de Floresta Ombrófila Mista Montana (Bioma Araucária) e plantação de Pinus elliotti Engelmann, no Parque Ecológico Vivat Floresta, Tijucas do Sul, Paraná, Brasil. Revista Brasileira de Zoologia 23(4):1159-1167.

GotelLi, N. J. \& ColWELL, R. K. 2001. Quantifying biodiversity: procedures and pitfalls in the measurement and comparison of species richness. Ecology Letter 4:379-391.

Guedes, R. S.; Zanella, F. C. V.; Costa Júnior, J. E. V.; SAntana, G. M. \& SiLVA, J. A. 2012. Caracterização florístico-fitossociológica do componente lenhoso de um trecho de caatinga no semiárido paraibano. Revista Caatinga 25(2):99-108.

Gullan, P. J. \& Cranston, P. S. 2012. Os insetos: um resumo de entomologia. São Paulo, Roca. 479p.

Gullan, P. J. \& Cranston, P. S. 2014. The insects: an outline of entomology. 5ed. Chichester, Wiley-Blackwell. 624p.

HernándeZ, M. I. M. 2005. Besouros Scarabaeidae (Coleoptera) da área do Curimataú, Paraíba. In: Araújo, F. S.; Rodal, M. J. N. \& Barbosa, M. R. V. eds. Análise das Variações da Biodiversidade do Bioma Caatinga: Suporte a Estratégias Regionais de Conservação. Brasília, Ministério do Meio Ambiente, p. 369-380.

Hernández, M. I. M. 2007. Besouros escarabeineos (Coleoptera: Scarabaeidae) da caatinga paraibana, Brasil. Oecologia brasiliensis 11(3):356-364.

Iannuzzi, L.; Maia, A. C. D.; Nobre, C. E. B.; Suzuki, D. K. \& Muniz, F. J. A. 2003. Padrões locais de Diversidade de Coleoptera (Insecta) em vegetação de Caatinga. In: Leal, I. R.; Tabarelli, M. \& Silva, J. M. C. eds. Ecologia e Conservação da Caatinga. Recife, Editora Universitária da UFPE, p. 367-389. 
Iannuzzi, L.; Maia, A. C. D. \& Vasconcelos, S. D. 2006. Ocorrência e sazonalidade de coleópteros buprestídeos em uma região de caatinga nordestina. Biociências 14(2): 174-179.

JimÉNEZ-VALVERdE, A. \& Hortal, J. 2003. Las curvas de acumulación de especies y la necesidad de evaluar la calidad de los inventarios biológicos. Revista Ibérica de Aracnología 8:151-161.

Lawrence, J. F. \& Britton, E. B. 1991. Coleoptera. In: NaumanN, I. ed. The Insects of Australia. A textbook for students and research workers. v.2. Melbourne, Melbourne University (CSIRO), p. 543-683.

Leal, I. R.; Silva, J. M. C.; Tabarelli, M. \& Lacker JR, T. E. 2005. Mudando o curso da conservação da biodiversidade na Caatinga do nordeste do Brasil. Megadiversidade 1(1):139-146.

LEWINSOHN, T. 2000. Avaliação do estado do conhecimento da diversidade biológica do Brasil. Brasília, MMA-GTB/CNPq-NEPA/UNICAMP. $520 \mathrm{p}$.

Liberal, C. N.; Farias, A. M. I.; Meiado, M. V.; Filgueiras, B. K. C. \& IANNUZZI, L. 2011. How habitat change and rainfall affect dung beetle diversity in Caatinga, a Brazilian semi-arid ecosystem. Journal of Insect Science 11(114):1-11.

Lima, R. L. L.; Andreazze, R.; Andrade, H. T. A. \& Pinheiro, M. P. G. 2010. Riqueza de Famílias e Hábitos Alimentares em Coleoptera Capturados na Fazenda da EMPARN-Jiqui, Parnamirim/RN. EntomoBrasilis 3(1): 11-15.

Linzmeier, A. M.; Ribeiro-Costa, C. S. \& MARinoni, R. C. 2006. Fauna de Alticini (Newman) (Coleoptera, Chrysomelidae, Galerucinae) em diferentes estágios sucessionais na Floresta com Araucária do Paraná, Brasil: diversidade e estimativa de riqueza de espécies. Revista Brasileira de Entomologia 50(1):101-109.

LOPES, P. P. \& LouZADA, J. N. C. 2005. Besouros (Coleoptera: Scarabaeidae e Histeridae). In: Junca, F. A.; Funch, L. S. \& Rocha, W. eds. Biodiversidade e Conservação da Chapada Diamantina. Brasília, Ministério do Meio Ambiente, p. 284-298.

Lopes, P. P.; LouZADA, J. N. C. \& VAZ-DE-Mello, F. Z. 2006. Organization of dung beetle communities (Coleoptera, Scarabaeidae) in areas of vegetation reestablishment in Feira de Santana, Bahia, Brazil. Sitientibus Série Ciências Biológicas 6(4):261-266.

Maia, A. C. D.; Iannuzzi, L.; Nobre, C. E. B. \& Albuqueroue, C. M. R. 2003. Padrões locais de diversidade de Cerambycidae (Insecta, Coleoptera) em vegetação de caatinga. In: LeaL, I. R.; TABARELLI, M. \& SiLva, J. M. C. eds. Ecologia e Conservação da Caatinga. Recife, Ed. Universitária da UFPE, p. 391-433.

Marinoni, R. C.; Ganho, N. G.; Monné, M. L. \& Mermudes, J. R. M. 2001. Hábitos alimentares em Coleoptera (Insecta). Ribeirão Preto, Holos Editora Ltda. 63p.

Marinoni, R. C. \& Dutra, R. R. C. 1997. Famílias de Coleoptera capturadas com armadilha Malaise em oito localidades do Estado do Paraná, Brasil. Diversidades alfa e beta. Revista Brasileira de Zoologia 14(3):751-770.

Mayer, A. C. G. \& Vasconcelos, S. D. 2013. Necrophagous beetles associated with carcasses in a semi-arid environment in Northeastern Brazil: Implications for Forensic Entomology. Forensic Science International 226:41-45.

Monné, M. L, \& Costa, C. 2019. Coleoptera in Catálogo Taxonômico da Fauna do Brasil. PNUD. Disponível em: <http://fauna.jbrj.gov. $\mathrm{br} /$ fauna/faunadobrasil/223>. Acesso em: $19 \mathrm{Fev} .2019$

Monkós, F. 1956. Sur le genere Megamerus MacLeay (Col. Chrysomelidae). Revue Francaise d'Entomologie 23:104-115.

Nascimento, F. E. L. \& Bravo, F. 2014. Espécies de Cerambycidae (Coleoptera) coletadas nas expedições do PPBio Semiárido. In: CALOR, A. R. \& Bravo, F. eds. Artrópodes do semiárido: biodiversidade e conservação. Feira de Santana, Printmídia, p. 127-138.

Nascimento, F. E. L.; Ferreira, A. S. \& Bravo, F. 2017. Cerambycidae (Coleoptera) do Semiárido: ampliando o conhecimento. In: Bravo, F. ed. Artrópodes do Semiárido II: Biodiversidade e conservação. 1 ed. São Paulo, Métis Produção Editorial, p. 76-98.

Nascimento, F. E. L.; Bravo, F. \& Monné, M. A. 2016. Cerambycidae (Insecta: Coleoptera) of Quixadá, Ceará State, Brazil: new records and new species. Zootaxa 4161(3):399-411.
NIMER, E. 1972. Climatologia da região Nordeste do Brasil. Introdução à climatologia dinâmica. Revista Brasileira de Geografia 34(2):3-51.

MonnÉ, M. L. \& Costa, C. 2018. Coleoptera in Catálogo Taxonômico da Fauna do Brasil. PNUD. Disponível em $<$ http://fauna.jbrj.gov.br/ fauna/faunadobrasil/223>. Acesso em $20 \mathrm{fev} 2018$.

Morse, G. 2014. Bruchidae Latreille, 1802. In: LesChEN, R. A. B. \& BeUTEL, R. G. eds. Handbook of Zoology: Morphology and Systematics (Chrysomeloidea, Curculionoidea). Vol. 3. Berlin, De Gruyter, p. 189-196.

Oksanen, J.; Blanchet, F. G.; Kindt, R.; Legendre, P.; Minchin, P. R.; O’Hara, R. B.; Simpson, G. L.; Solymos, P.; Stevens, M. H. H. \& WAGNer, H. 2016. vegan: Community Ecology Package. R package version 2. 3-5.

Prado, D. 2003. As caatingas da América do Sul. In: Leal, I. R.; Tabarelli, M. \& SiLVA, J. M. C. eds. Ecologia e conservação da Caatinga. Recife, Editora Universitária da Universidade Federal de Pernambuco, p. 3-73.

R Core TEAm. 2018. R: A language and environment for statistical computing. Vienna, R Foundation for Statistical Computing, 2018. Disponível em <http://www.R-project.org>. Acesso em 26 mar 2018.

Ribeiro-Costa, C. S. \& Almeida, L. M. 2012. Seed-Chewing Beetles (Coleoptera: Chrysomelidae: Bruchinae). In: PAnizzI, A. R. \& PARRA, J. R. P. eds. Insect Bioecology and Nutrition for Integrated Pest Management. Boca Raton, CRC Press, p. 325-352.

SALOMÃo, R. P. \& IANNUZZI, L. 2015. Dung beetle (Coleoptera, Scarabaeidae) assemblage of a highly fragmented landscape of Atlantic forest: from small to the largest fragments of northeastern Brazilian region. Revista Brasileira de Entomologia 59(2):126-131.

Salomão, R. P.; Lira, F. A. \& Iannuzzi, L. 2014. Dominant dung beetle (Coleoptera: Scarabaeidae: Scarabaeinae) species exhibit wider trophic niches on fruits, excrement, and carrion in Atlantic Forest, Brazil. The Coleopterists Bulletin 68(4):686-688.

Santos, J. C.; Leal, I. R.; Almeida-Cortez, J. S.; Fernandes, G. W. \& TABARELli, M. 2011. Caatinga: the scientific negligence experienced by a dry tropical forest. Tropical Conservation Science 4(3):276-286.

SANTOS, D. S. \& SANTOS, W. E. 2016. Association of necrophilous beetles (Coleoptera) with rat carcasses in a semi-arid area, Northeastern Brazil. Entomotropica 31(2):167-171.

Santos, W. E.; Alves, A. C. F. \& Creão-Duarte, A. J. 2014. Beetles (Insecta, Coleoptera) associated with pig carcasses exposed in a Caatinga area, Northeastern Brazil. Brazilian Journal of Biology 74(3):649-655.

Silva, J. M. C.; Barbosa, L. C. F.; Leal, I. R. \& Tabarelli, M. 2017. The Caatinga: Understanding the Challenges. In: SiLva, J. M. C.; Leal, I. R. \& Tabarelli, M. eds. Caatinga: The Largest Tropical Dry Forest Region in South America. Cham, Springer International Publishing, p. 1-19.

Silva, J. M. C.; Tabarelli, M. \& Fonseca, M. T. 2003. Áreas e ações prioritárias para a conservação da biodiversidade da Caatinga. In: Silva, J. M. C.; Tabarelli, M.; Fonseca, M. T. \& Lins, L. V. eds. Biodiversidade da Caatinga: áreas e ações prioritárias para a conservação. Brasília, Ministério do Meio Ambiente, p. 349-374.

TABARELl, M. \& Silva, J. M. C. 2003. Áreas e ações prioritárias para a conservação da biodiversidade da caatinga. In: LEAL, I. R.; TABARELLI, M. \& SILVA J. M. C. eds. Ecologia e Conservação da Caatinga. Recife, Editora Universitária, p. 777-796.

Triplehorn, C. A. \& Johnson, N. F. 2011. Estudo dos Insetos. São Paulo, Cengage Learning. 809p.

Vasconcellos, A.; Andreazze, R.; Almeida, A. M.; AraúJo, H. F. P.; Oliveira, E. S. \& Oliveira, U. 2010. Seasonality of insects in the semi-arid Caatinga of the northeastern Brasil. Revista Brasileira de Entomologia 3:471-476

Velloso, A. L. Sampaio, E. V. S. B. \& Pareyn, F. G. C. 2002. Ecorregiões propostas para o bioma caatinga. Recife, Associação Plantas do Nordeste, Instituto de Conservação Ambiental, The Nature Conservancy do Brasil. 75p.

VieIRA, L.; SiLVA, F. A. B. \& Louzada, J. 2017. Dung beetles in a Caatinga Natural Reserve: a threatened Brazilian dry-forest with high biological value. Iheringia, Série Zoologia 107:e2017045. 